\title{
Utilization Statistics based Optimal Assignment of Wavelength Converters with Self Adaptive Differential Evolution
}

\author{
Ramya Shivamadegowda and Indumathits \\ ${ }^{1}$ Dept. of Electronics \& Communication Engineering \\ R.V.College of Engineering \\ Bengaluru, India \\ ${ }^{2}$ Dept. of Digital electronics and communication system \\ V.I.A.T, V.T.U \\ Mudenahalli, Chickaballapur district, India \\ ramyas@rvce.edu.in,indumathi_ts@yahoo.co.in
}

\begin{abstract}
In this work, optimal assignment of full range wavelength converters have done over available nodes in wavelength division multiplexing optical network to maximize the total utilization of deployed converters. This will increase the performance by cost ratio by keeping the deployed converters busy maximally. The optimization model has solved by heuristic method to search the solution space efficiently through self adaptive form of differential evolution. A mechanism has also proposed to handle the dynamic utilization under the same network through maintaining the diversity in the population by insertion of a new member in each generation.
\end{abstract}

Keywords: WDM optical networks, optimal wavelength converter allocation, Differential evolutionary algorithm, Utilization matrix, Wavelength converter allocation matrix

\section{Introduction}

Wavelength Division Multiplexing (WDM) technique utilizes the big information measure of glass fibre by overcoming the electronic bottleneck of slow switches.WDM optical networks utilize light weight methods to exchange info between source-destination node-pairs. A light path is associate all optical continuous channels established between a node-pair. Establishment of light path with pre-information of connection nodes through some algorithmic approach is called as Routing and Wavelength Assignment (RWA). A wavelength device is a associate device that transforms a wavelength to a distinct wavelength. If no wavelength device is employed, a light path established between a sources destination node-pair continuously consists of hops of same wavelength. When this wavelength continuity constraint cannot be meet, the light path is blocked. Either all or restricted range of nodes in a very WDM optical network could use wavelength converters to extend the traffic-carrying capability by quiet the wavelength continuity constraint. betting on conversion vary, the wavelength converters may be classified into 2 types: Full vary Wavelength device (FWC) that may convert associate input wavelength to any possible wavelength and Limited-range Wavelength device (LWC) that the conversion capability is proscribed to a set of potential wavelengths, however, available with lower price. During this paper, we've applied the consideration of FWCs.The improved performance because of the incorporation of wavelength converters is restricted and supported mostly by the constellation still because the traffic configuration. in a very tiny network with comfortable wavelength channels and underneath lightweight traffic, it

Received (May 10, 2017), Review Result (August 30, 2017), Accepted (September 8, 2017) 
wants no wavelength converters in any respect because the chance of wavelength conflict is little. Even underneath significant traffic, the performance improvement is also insignificant because the network might not have comfortable free channels to ascertain a light-weight path. Underneath moderate network size and medium traffic load, it's continuously advantageous to use wavelength converters to keep up the obstruction chance to a lower level; however, the value of planning WDM optical networks goes high because of the incorporation of wavelength converters. But, because of the irregular topology of all-optical wide space network, few nodes could need less range of converters. Moreover, there is also some nodes that don't need any device in any respect. Therefore, best Allocation of Wavelength Converters (OWCA) may be a difficult and fascinating sensible issue.

\section{Related Work}

Taking into account the requirements to the converters e.g., bit rate transparency, polarization independence, wavelength independence, moderate input power levels, high signal-to-noise ratio and high extinction ratio interferometric wavelength convertors are very interesting for use in WDM optical fibre networks[1].The wavelength continuity restriction obligatory by WDM network ends up in not proper utilization of wavelength channels and high obstruction chance with poor block performance in WDM transport networks. So as to weaken the affinity of the wavelength continuity constraint, there is need of wavelength converter and wavelength rerouting. Thought of the price, wavelength rerouting methodology could be a sensible technique which supported the model of wavelength graph, the wavelength rerouting strategy is planned [2].[3] Provided the analysis of the impact of spectrum conversion on the lightpath obstruction performance within the CO-OFDM optical networks. The performance of wavelength division multiplexing (WDM) networks is very hooked in to the wavelength device allocation and routing issues. Typically, these issues are treated as freelance issues underneath dynamic traffic; but, so as to attain sensible system's performance, each issue ought to be treated along. To this aim [4] has proposed a joint optimization approach where allocations for converters and paths for routing are calculated simultaneously. Based on different parameters like number of channels, associated wavelength and required number of hopes between nodes performances of WDM have been discussed in [5].The benefits of converters in WDM have discussed in [6].To maximize the performance, it is necessary to allocate wavelength converters to the network nodes optimally.[ 7] has proposed a multiobjective optimization approach, based on a multi-objective evolutionary algorithm (MOEA), where the blocking probability and the number of wavelength converters are simultaneously minimizing.[8] has proposed the solution for two important challenges of how many optimal wavelength converters required, which should be minimum as much as possible and where they have to place so that required success in blocking probability could be achieved efficiently. Both challenges have handled simultaneously with concept of evolutionary computation which includes elitism concept and a variation of genetic algorithm.[9] has reviewed silicon photonic circuits for multiple-channel modulators, polarization-insensitive WDM receiver, and variable optical attenuators with multiplexer. In [10] changes on Optical Signal-to-Noise Ratio (OSNR) are observed by changing the number of channels on the transmission line, channel spacing, noise modulation technique and output power. In [11] mentioned the non-blocking multicast RWA downside in 2 phases with relevancy the cascaded combination of associate AWG-based broadcast Clos network, referred to as copy network, and a point-to-point AWG-based Clos network.

\section{Problem Statement}

In this section, we have a tendency to describe the optimization downside into account and therefore the corresponding mathematical formulation. 
The following notations are used: $N=$ Number of nodes in the WDM optical network.

$M i=$ Number of FWCs required at node $i$ for complete wavelength conversion.

$M=$ Maximum value of the set $\{M 1, M 2, \ldots, M N\}$.

$T=$ Total number of available FWCs.

$\boldsymbol{U}=$ Utilization matrix (of $\operatorname{order} N \times(M+1)$ ), in which the $(i, j)$-th entry (where, $1 \leq i \leq N, 0 \leq j \leq M$ ) denotes the percentage of time that $j$ FWCs are being utilized simultaneously at node $i$. So if node $i$ has $J$ wavelength converters, the utilizations at node $i$ is

$$
\sum_{j=1}^{J} U_{i, j}
$$

Given the utilization matrix, $\boldsymbol{U}$, it needs to determine the wavelength converter allocation matrix, $\boldsymbol{x}$ (of order $N \times M$ ) where,

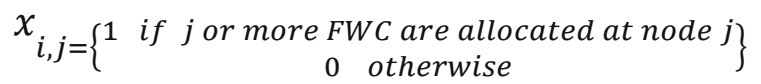

Accordingly, the total utilizations at node $i$ is

$$
\sum_{j=1}^{M} U_{i, j} x_{i, j}
$$

The objective function can be formulated as Maximize the sum of total utilizations

$$
\sum_{i=1}^{N} \sum_{j=1}^{M} U_{i, j} x_{i, j}
$$

Subjected to the following constraints:

$$
\sum_{i=1}^{N} \sum_{j=1}^{M} x_{i, j}=\mathrm{T} ; x_{i, j} \geq x_{i, j+1}
$$

\section{Performance Evaluation}

Network NSFNET T1 as shown in Figure 1 has considered in this work .In this network there are 14 nodes and 21 bi-directional links available.

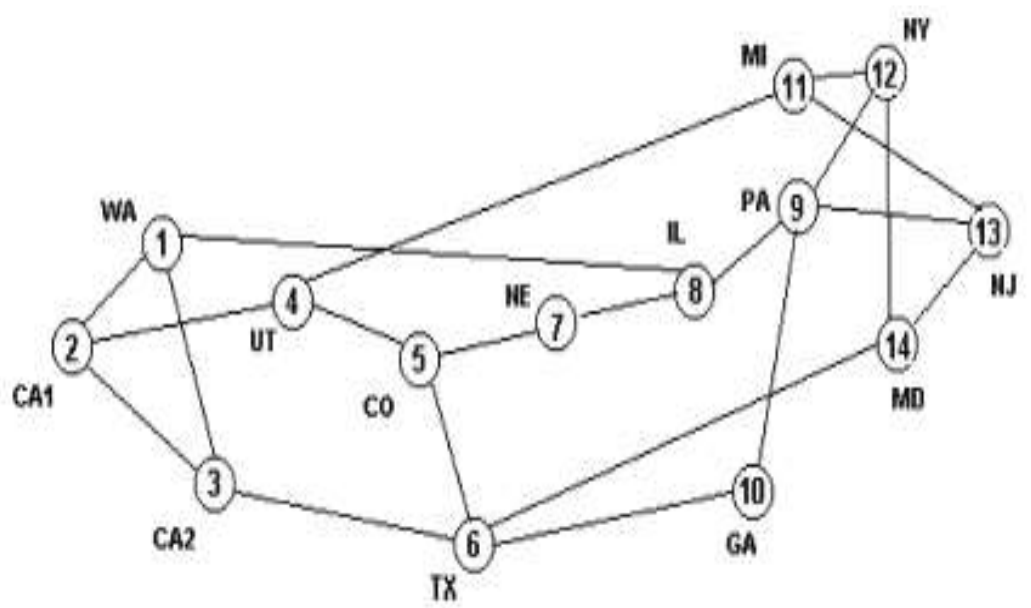

Figure 1. NSFNET T1 Backbone Network

The utilization matrix corresponding to selected network has shown in Table 1. It is clear that because of six columns in utilization matrix, the required value of $M$ is equal to 5.Three different possibilities of available total number of convertors $(18,20,24)$ have explored with this network. 
Table 1. Utilization Matrix, $U 1$

\begin{tabular}{|l|l|l|l|l|}
\hline 0.44 & 0.41 & 0.13 & 0.02 & 0.00 \\
\hline 0.41 & 0.38 & 0.16 & 0.03 & 0.02 \\
\hline 0.52 & 0.44 & 0.03 & 0.01 & 0.00 \\
\hline 0.11 & 0.51 & 0.22 & 0.10 & 0.04 \\
\hline 0.64 & 0.25 & 0.07 & 0.04 & 0.00 \\
\hline 0.59 & 0.32 & 0.09 & 0.00 & 0.00 \\
\hline 0.12 & 0.54 & 0.22 & 0.07 & 0.03 \\
\hline 0.47 & 0.43 & 0.08 & 0.01 & 0.01 \\
\hline 0.71 & 0.21 & 0.08 & 0.00 & 0.00 \\
\hline 0.21 & 0.57 & 0.12 & 0.04 & 0.03 \\
\hline 0.46 & 0.38 & 0.13 & 0.03 & 0.01 \\
\hline 0.39 & 0.51 & 0.09 & 0.01 & 0.00 \\
\hline 0.35 & 0.43 & 0.12 & 0.09 & 0.01 \\
\hline 0.67 & 0.28 & 0.03 & 0.02 & 0.00 \\
\hline
\end{tabular}

\section{Proposed Approach}

An optimization model has applied to define the location of converters so that total utilization could be maximized. This approach will place converters with nodes as according to need with the nodes. The searching capability of differential evolution has applied to define the position of different number of convertors at the nodes. There is high probability to achieve the global solution efficiently by differential evolution approaches. A self adaptive version of differential evolution, in which control parameters have obtained the value adaptively. The proposed algorithm explore the possible solution through transforming the real number of solution in to binary domain under define objective function and constraints. A dynamic condition has been created in the simulation by vary the utilization matrix with time. To handle such condition in each generation a new solution is embedded in replacement of weakest solution. This approach will maintain the diversity and provide help in finding new solution when there is new utilization matrix appeared.

The differential evolution (DE) these days is taken jointly of sturdy entity beneath natural process computation to unravel global optimization issues.Functionally, a trial vector is created through the differential from the available other member in the population as shown in Eq.5.There are various possibilities exist in terms of who will form this differential form in this work, approach $\mathrm{DE} / \mathrm{rand} / 1$ has been applied. The crossover operator has applied between parent and the corresponding trial vector under the probabilitistic environment as shown in Eq.6.The CR parameter takes value in the range of [0 1].To define a minimum single position crossover ,forced crossover through jrand,which has to select a number in the range of [1 NP] has taken. Selection between parent and offspring is decide through the greedy selection i.e. who is more fitter will join the next generation and other will die as given in Eq.7.To define the self adaptiveness of control parameters like $\mathrm{F}$ and $\mathrm{CR}$, a self adaptive approach has given in Eq.8. and Eq.9.The quantities randj, $\mathrm{j} \epsilon$ represent uniform random values at intervals the vary $[0$, 1]. $\tau 1$ and $\tau 2$ works under probalistic environment to regulate management parameters $F$ and $\mathrm{Cr}$, severally. Constants $\tau 1, \tau 2, \mathrm{Fl}, \mathrm{Fu}$ assigned fastened values to $0.1,0.1,0.1,0.9$ severally. The management parameter values decide before the mutation operation has to define.

$$
V_{i}^{(G)}=X_{r 1}^{(G)}+F *\left(X_{r 2}^{(G)}-X_{r 3}^{(G)}\right)
$$




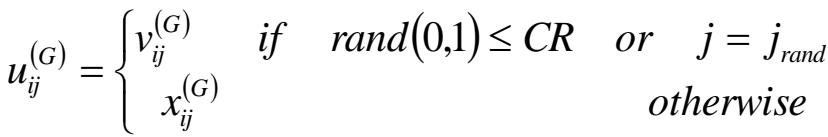

$$
\begin{aligned}
& x_{i j}^{(G)}=\left\{\begin{array}{lrr}
u_{i}^{(G)} & \text { if } & f\left(u_{i}^{(G)}\right) \leq f\left(x_{i}^{(G)}\right) \\
x_{i}^{(G)} & & \text { otherwise }
\end{array}\right. \\
& F_{i}^{(G+1)}=\left\{\begin{array}{cc}
F_{1}+\operatorname{rand}_{1} * F_{u} & \text { if } \text { rand }_{2}<\tau_{1} \\
F_{i}^{(G)} & \text { otherwise }
\end{array}\right.
\end{aligned}
$$

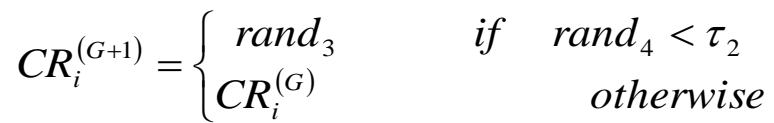

\section{Experimental Results}

The mathematical model and algorithms have been implemented under MATLAB environment. The population size of 100 has taken in both algorithms while there are total 500 number of iterations have allowed for final convergence. With the three cases of available number of FWC which are 18, 20 and 24 both DE \& SADE have been evolved. The obtained allocations for different cases have shown in Case 1, Case 2 and Case 3.For all the three cases the utilization matrix U1 as shown in Table 1 has applied .The obtained allocations for all the three different cases have shown in Table 2 to Table 4.The obtained value of utilization to each case has also shown in Table 5.Comparative convergence between DE and SADE have been shown in Figure 2to Figure 4. In all cases, the utilization value is same but it is clear that SADE has faster convergence in compare to $\mathrm{DE}$ in all cases. At the beginning of convergence graph there are some negative value has come .This is because of penalty concept has applied to make the solution feasible under constraint environment. To define the dynamic environment in Case 4 two different utilization matrix has applied. Utilization matrix (U1) has applied if iteration is less than 300 iterations, if iteration is more than 300 , a second utilization matrix (U2) as shown in Table 6 have applied. It is clear that two different allocations have appeared as shown in Table 7 with their utilization value in Table8. Convergence characteristic corresponding to dynamic environment has shown in Figure 5. It is clear that within few iteration with new utilization matrix solution has been converge.

\section{Case 1: Available No. of FWC $=18$}

\begin{tabular}{|c|c|c|c|c|c|c|c|c|c|c|}
\hline \multirow{2}{*}{$\begin{array}{c}\text { Nodes(18) } \\
1\end{array}$} & \multicolumn{5}{|c|}{ Placed Converters(DE) } & \multicolumn{5}{|c|}{ Placed Converters(SADE) } \\
\hline & 1 & 1 & 0 & 0 & 0 & 1 & 1 & 0 & 0 & 0 \\
\hline 2 & 1 & 1 & 0 & 0 & 0 & 1 & 1 & 0 & 0 & 0 \\
\hline 3 & 1 & 0 & 0 & 0 & 0 & 1 & 0 & 0 & 0 & 0 \\
\hline 4 & 1 & 1 & 0 & 0 & 0 & 1 & 1 & 0 & 0 & 0 \\
\hline 5 & 1 & 0 & 0 & 0 & 0 & 1 & 0 & 0 & 0 & 0 \\
\hline 6 & 1 & 0 & 0 & 0 & 0 & 1 & 0 & 0 & 0 & 0 \\
\hline 7 & 1 & 1 & 0 & 0 & 0 & 1 & 1 & 0 & 0 & 0 \\
\hline 8 & 1 & 0 & 0 & 0 & 0 & 1 & 0 & 0 & 0 & 0 \\
\hline 9 & 1 & 0 & 0 & 0 & 0 & 1 & 0 & 0 & 0 & 0 \\
\hline 10 & 1 & 0 & 0 & 0 & 0 & 1 & 0 & 0 & 0 & 0 \\
\hline 11 & 1 & 0 & 0 & 0 & 0 & 1 & 0 & 0 & 0 & 0 \\
\hline 12 & 1 & 0 & 0 & 0 & 0 & 1 & 0 & 0 & 0 & 0 \\
\hline 13 & 1 & 0 & 0 & 0 & 0 & 1 & 0 & 0 & 0 & 0 \\
\hline 14 & 1 & 0 & 0 & 0 & 0 & 1 & 0 & 0 & 0 & 0 \\
\hline
\end{tabular}

Table 2. FWC Allocation with $\mathrm{T}=18$ by DE \&SADE 


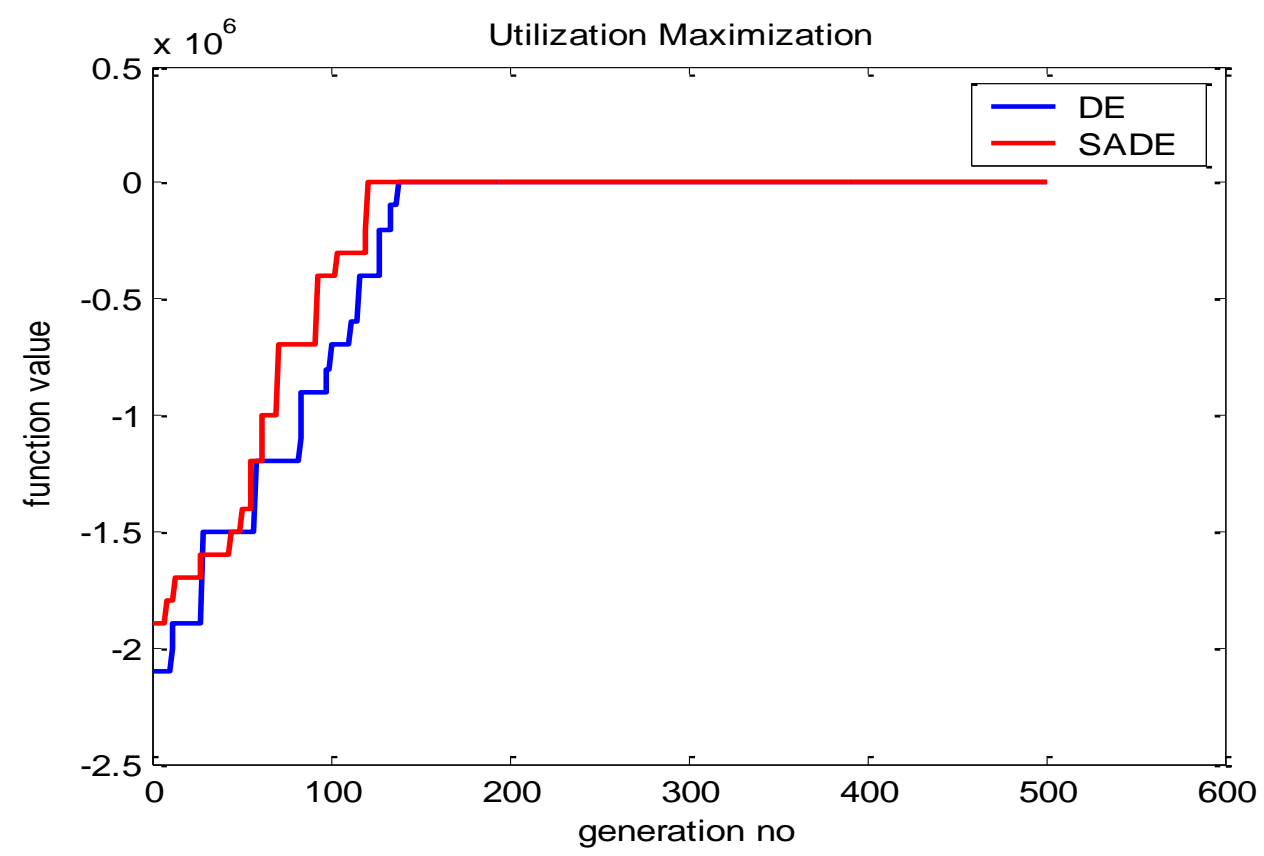

Figure 2. Convergence Characteristic of DE \& SADE with $T=18$

Case 2: Available No.of FWC $=20$

Table 3. FWC Allocation with $\mathrm{T}=20$ by DE \&SADE

\begin{tabular}{|c|c|c|c|c|c|c|c|c|c|c|}
\hline \multirow{2}{*}{$\frac{\operatorname{Nodes}(\mathbf{2 0})}{1}$} & \multicolumn{5}{|c|}{ Placed Converters(DE) } & \multicolumn{5}{|c|}{ Placed Converters(SADE) } \\
\hline & 1 & 1 & 0 & 0 & 0 & 1 & 1 & 0 & 0 & 0 \\
\hline 2 & 1 & 1 & 0 & 0 & 0 & 1 & 1 & 0 & 0 & 0 \\
\hline 3 & 1 & 0 & 0 & 0 & 0 & 1 & 0 & 0 & 0 & 0 \\
\hline 4 & 1 & 1 & 0 & 0 & 0 & 1 & 1 & 0 & 0 & 0 \\
\hline 5 & 1 & 0 & 0 & 0 & 0 & 1 & 0 & 0 & 0 & 0 \\
\hline 6 & 1 & 0 & 0 & 0 & 0 & 1 & 0 & 0 & 0 & 0 \\
\hline 7 & 1 & 1 & 0 & 0 & 0 & 1 & 1 & 0 & 0 & 0 \\
\hline 8 & 1 & 0 & 0 & 0 & 0 & 1 & 0 & 0 & 0 & 0 \\
\hline 9 & 1 & 0 & 0 & 0 & 0 & 1 & 0 & 0 & 0 & 0 \\
\hline 10 & 1 & 1 & 0 & 0 & 0 & 1 & 0 & 0 & 0 & 0 \\
\hline 11 & 1 & 1 & 0 & 0 & 0 & 1 & 1 & 0 & 0 & 0 \\
\hline 12 & 1 & 0 & 0 & 0 & 0 & 1 & 0 & 0 & 0 & 0 \\
\hline 13 & 1 & 0 & 0 & 0 & 0 & 1 & 1 & 0 & 0 & 0 \\
\hline 14 & 1 & 0 & 0 & 0 & 0 & 1 & 0 & 0 & 0 & 0 \\
\hline
\end{tabular}




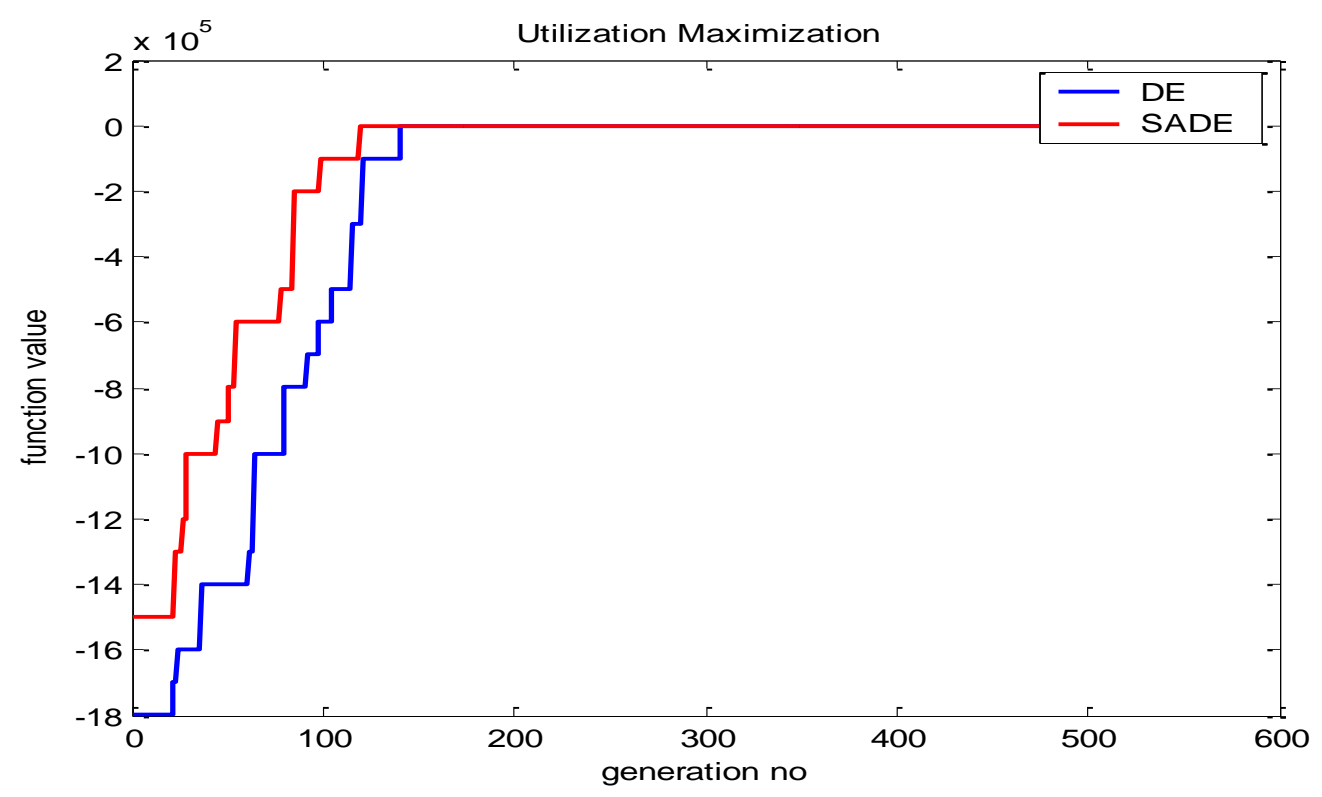

Figure 3. Convergence Characteristic of DE \& SADE with $\mathrm{T}=\mathbf{2 0}$

Case 3: Available No. of $\mathrm{FWC}=24$

Table 4. FWC Allocation with $\mathrm{T}=24$ by DE \&SADE

\begin{tabular}{|c|c|c|c|c|c|c|c|c|c|}
\hline \multirow{2}{*}{$\frac{\operatorname{Nodes}(\mathbf{2 4})}{1}$} & \multicolumn{4}{|c|}{ Placed Converters(DE) } & \multicolumn{5}{|c|}{ Placed Converters(SADE) } \\
\hline & $\begin{array}{ll}1 & 1\end{array}$ & 0 & 0 & 0 & 1 & 1 & 0 & 0 & 0 \\
\hline 2 & 1 & 0 & 0 & 0 & 1 & 1 & 0 & 0 & 0 \\
\hline 3 & 1 & 0 & 0 & 0 & 1 & 0 & 0 & 0 & 0 \\
\hline 4 & 1 & 1 & 0 & 0 & 1 & 1 & 1 & 0 & 0 \\
\hline 5 & 1 & 0 & 0 & 0 & 1 & 0 & 0 & 0 & 0 \\
\hline 6 & 1 & 0 & 0 & 0 & 1 & 1 & 0 & 0 & 0 \\
\hline 7 & 1 & 0 & 0 & 0 & 1 & 1 & 0 & 0 & 0 \\
\hline 8 & 1 & 0 & 0 & 0 & 1 & 0 & 0 & 0 & 0 \\
\hline 9 & 1 & 0 & 0 & 0 & 1 & 0 & 0 & 0 & 0 \\
\hline 10 & 1 & 0 & 0 & 0 & 1 & 1 & 0 & 0 & 0 \\
\hline 11 & 1 & 0 & 0 & 0 & 1 & 1 & 0 & 0 & 0 \\
\hline 12 & 11 & 0 & 0 & 0 & 1 & 1 & 0 & 0 & 0 \\
\hline 13 & 1 & 0 & 0 & 0 & 1 & 1 & 0 & 0 & 0 \\
\hline 14 & 10 & 0 & 0 & 0 & 1 & 0 & 0 & 0 & 0 \\
\hline
\end{tabular}

Table 5. Utilization Value Obtained with Different Number of FWC

\begin{tabular}{|c|c|c|c|}
\hline No. of FWC & T=18 & T=20 & T=24 \\
\hline Utilization value & 6.39 & 6.64 & 7.04 \\
\hline
\end{tabular}




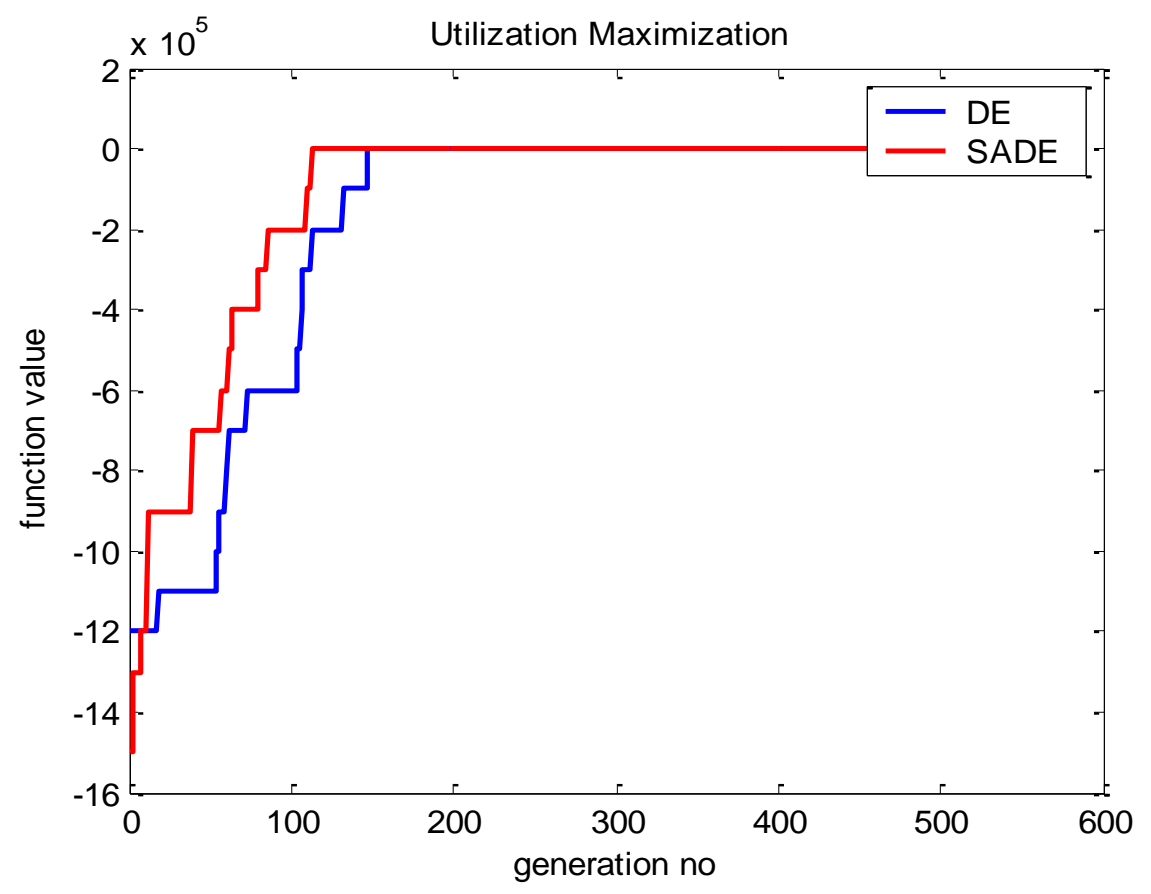

Figure 4. Convergence Characteristic of DE \& SADE with $T=24$

Case 4: Available No. of $\mathrm{FWC}=18$ in dynamic environment

Table 6. Utilization Matrix (U2)

\begin{tabular}{|l|l|l|l|l|}
\hline 0.50 & 0.43 & 0.05 & 0.02 & 0.00 \\
\hline 0.41 & 0.36 & 0.18 & 0.05 & 0.00 \\
\hline 0.50 & 0.44 & 0.05 & 0.01 & 0.00 \\
\hline 0.11 & 0.51 & 0.27 & 0.05 & 0.04 \\
\hline 0.60 & 0.25 & 0.11 & 0.04 & 0.00 \\
\hline 0.45 & 0.40 & 0.09 & 0.06 & 0.00 \\
\hline 0.12 & 0.54 & 0.22 & 0.07 & 0.03 \\
\hline 0.37 & 0.43 & 0.13 & 0.06 & 0.01 \\
\hline 0.71 & 0.21 & 0.08 & 0.0 .0 & 0.00 \\
\hline 0.21 & 0.57 & 0.12 & 0.04 & 0.03 \\
\hline 0.46 & 0.38 & 0.13 & 0.03 & 0.01 \\
\hline 0.39 & 0.51 & 0.09 & 0.01 & 0.00 \\
\hline 0.30 & 0.43 & 0.17 & 0.09 & 0.01 \\
\hline 0.60 & 0.28 & 0.10 & 0.02 & 0.00 \\
\hline
\end{tabular}

Table 7. FWC Allocation by SADE in Dynamic Environment with $\mathrm{T}=18$

\begin{tabular}{|c|c|c|c|c|c|c|c|c|c|c|}
\hline \multirow{2}{*}{$\begin{array}{c}\text { Nodes }(\mathbf{1 8}) \\
1\end{array}$} & \multicolumn{5}{|c|}{ Placed Converters(SADE) } & \multicolumn{5}{|c|}{ Placed Converters(SADE) } \\
\hline & 1 & 1 & 0 & 0 & 0 & 1 & 0 & 0 & 0 & 0 \\
\hline 2 & 1 & 1 & 0 & 0 & 0 & 1 & 1 & 0 & 0 & 0 \\
\hline 3 & 1 & 0 & 0 & 0 & 0 & 1 & 0 & 0 & 0 & 0 \\
\hline 4 & 1 & 1 & 0 & 0 & 0 & 1 & 1 & 0 & 0 & 0 \\
\hline 5 & 1 & 0 & 0 & 0 & 0 & 1 & 0 & 0 & 0 & 0 \\
\hline 6 & 1 & 0 & 0 & 0 & 0 & 1 & 0 & 0 & 0 & 0 \\
\hline 7 & 1 & 1 & 0 & 0 & 0 & 1 & 1 & 0 & 0 & 0 \\
\hline 8 & 1 & 0 & 0 & 0 & 0 & 1 & 0 & 0 & 0 & 0 \\
\hline 9 & 1 & 0 & 0 & 0 & 0 & 1 & 0 & 0 & 0 & 0 \\
\hline 10 & 1 & 0 & 0 & 0 & 0 & 1 & 0 & 0 & 0 & 0 \\
\hline
\end{tabular}




\begin{tabular}{|l|lllll|rrrrrr|}
\hline 11 & 1 & 0 & 0 & 0 & 0 & 1 & 1 & 0 & 0 & 0 \\
\hline 12 & 1 & 0 & 0 & 0 & 0 & 1 & 0 & 0 & 0 & 0 \\
\hline 13 & 1 & 0 & 0 & 0 & 0 & 1 & 0 & 0 & 0 & 0 \\
\hline 14 & 1 & 0 & 0 & 0 & 0 & 1 & 0 & 0 & 0 & 0 \\
\hline
\end{tabular}

Table 8. Utilization Value Obtained with U1 \&U2 with FWC=18

\begin{tabular}{|c|c|c|}
\hline Algorithm & U.Matrix(1) & U.Matrix(2) \\
\hline SADE & 6.3900 & 6.5400 \\
\hline
\end{tabular}

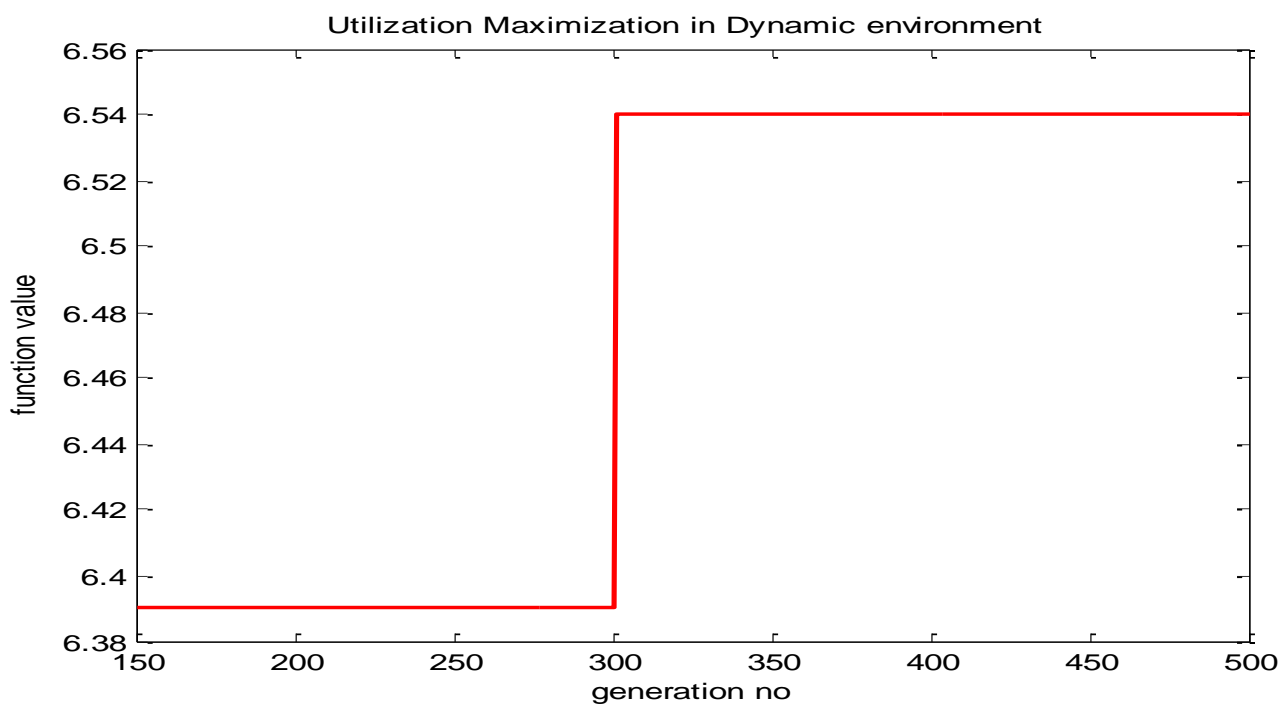

Figure 5. Convergence Characteristic of SADE with $\mathrm{T}=18$ in Dynamic

\section{Conclusion}

All-optical networks deliver information in the optical domain so that the electronic bottleneck can be avoided. They can support high data rate and provide large network capacity. In this work we focus on the problem of assigning the wavelength converters over different nodes having different traffic condition. To maximize the performance, it is necessary to allocate wavelength converters to the network nodes optimally. In this approach, utilization statistics of wavelength converters are collected from computer simulation, and then optimization algorithms are designed to allocate wavelength converters based on the utilization statistics. We have applied two varient of differential evolution one is with fixed parameters while other is self adaptive in parameters value. With various experimental analysis under different possibilities of available converters, optimal allocation have obtained under utilization statistics.Eventhough obtained utilization values are same for DE and SADE, but convergence rate is faster in the case of SADE. It is not necessary that utilization statistics is remain same with time hence a very fast re-establishment solution has presented to handle the dynamic environment of utilization statistics.

\section{References}

[1] S.L. Danielsen, B. Mikkelsen, P.B. Hansen, H.N. Poulsen and A. Kloch K.E.Stubkjaer, Wavelength conversion techniques and devices", Lasers and Electro-Optics Society Annual Meeting, LEOS, 97 10th Annual Meeting Conference proceedings, IEEE, (1997).

[2] T.-H. Zhou, M.-S. Chen, N. He, "Research on the Wavelength Rerouting Algorithm to Replace the Wavelength Convertor",Communications, Circuits and Systems Proceedings, (2006). 
[3] L. Peng, C.-H. Youn and C. Qiao, "Theoretical Analyses of Lightpath Blocking Performance in COOFDM Optical Networks with/without Spectrum Conversion”, IEEE Communications Letters, vol. 17, iss. 4, (2013).

[4] D.P. P into-Roa, B. Barán and C.A. Brizuela, "Routing and wavelength converter allocation in WDM networks: a multi-objective evolutionary optimization approach", Photon Netw Commun. doi:10.1007/s11107-011-0304-4, vol. 22, (2011), p. 23.

[5] M.K. Dutta and V.K. Chaubey, "Performance Analysis of All-Optical WDM Network with Wavelength Converter Using Erlang C Traffic Model”, In: Das V.V. (eds) Information Processing and Management. Communications in Computer and Information Science, Springer, Berlin, Heidelberg, vol 70, (2010).

[6] G. Xiao and Y.-W. Leung, "Allocation of Wavelength Converters in All-Optical Networks", Optical Networks, Volume 6 of the series Network Theory and Applications, (2001), pp 299-345.

[7] R. Maciel, "Optimal wavelength converter allocation: a new approach based MOEA", LANC '09 Proceedings of the 5th International Latin American Networking Conference, (2009), pp. 41-48.

[8] S Ramya and T S Indumathi, "Evolutionary design for optimal number of wavelength converters and their placement in WDM", Applied and Theoretical Computing and Communication Technology (iCATccT), (2015).

[9] P. Dong, "Silicon Photonic Integrated Circuits for Wavelength-Division Multiplexing Applications", IEEE Journal of Selected Topics in Quantum Electronics ( Volume: 22, Issue: 6, Nov.-Dec. 2016 )

[10] M. Yucel, M. S. Osmanca and H. Alparslan, "Performance measurement and analysis in WDM Networks effects on OSNR", Electrical and Electronic Engineering (ICEEE), (2017).

[11] M. Ge, T. Ye, T. T. Lee and W. Hu, "Multicast Routing and Wavelength Assignment in AWG-Based Clos Networks", IEEE/ACM Transactions on Networking, vol. 25, iss. 3, (2017). 